\title{
Computer-Assisted Image Analysis of Amyloid Deposits in Abdominal Fat Pad Aspiration Biopsies
}

Svetoslav Bardarov, M.D., Claire W. Michael, M.D., Robert T. Pu, M.D., Ph.D., and Yijun Pang, M.D., Ph.D. ${ }^{*}$

\begin{abstract}
Amyloidosis is a heterogeneous group of diseases with a common outcome: deposition of insoluble protein in the visceral organs and tissues. Primary amyloidosis is a consequence of different plasma cell disorders, and it is the most common form of amyloidosis in the United States with an estimated 2,000 new cases annually. Other forms of amyloidosis include chronic inflammatory processes, familial type of amyloidosis, and localized forms like Alzheimer's disease.

The diagnosis of amyloidosis is based on the clinical picture and demonstration of amyloid deposit in tissues with Congo-red stain. In our article, we describe a simple methodology for image analysis of fat pad biopsies for amyloidosis using a commercially available software Adobe Photoshop CS3C Extended Edition. The principle is based on calculation of the mean gray value of each blue and green channel and comparison of their ratios. As a negative control, we have used samples from heart, scar tissue, and skin with their representative control. Fibrous tissue often gives a white:blue to blue:green birefringence, which often is confused with the apple: green birefringence of the amyloid stain; however, we were successful in discriminating these colors using the methodology described in this article. We also analyzed 22 patients with at least 2 years follow-up in our institution. The specificity and the sensitivity of the computerassisted image analysis were calculated to be $75 \%$ and $100 \%$, respectively. These results are in agreement with the published papers (references here); however, caution should be exercised before drawing firm conclusions because of the small sample size presented here. Diagn. Cytopathol. 2009;37:30-35.

○ 2008 Wiley-Liss, Inc.
\end{abstract}

Key Words: image analysis; amyloid; abdominal fat pad

Department of Cytopathology, University of Michigan, Ann Arbor, Michigan

*Correspondence to: Yijun Pang, M.D., Ph.D., Department of Pathology, 1500 East Medical Drive, Ann Arbor, MI 48109.

E-mail: ypang@med.umich.edu

Received 4 June 2008; Accepted 30 July 2008

DOI 10.1002/dc.20948

Published online 29 October 2008 in Wiley InterScience (www. interscience.wiley.com).
Amyloidosis represents a group of diseases that results in abnormal insoluble protein deposition in soft tissues and solid organs. These abnormal proteins have different chemical structures; however, the secondary beta pleated sheet structures are similar. Initially amyloidosis as a disease was classified as primary, secondary, and familial, with an autonomic dominant pattern of inheritance. Primary amyloidosis is defined as deposition of immunoglobulin light chain deposition or less often the heavy chain deposits. Secondary amyloidosis is defined as a result of heterogeneous group of diseases such as chronic inflammatory disease, rheumatoid arthritis, or various infective etiologies. Currently, the amyloidosis classification is based on the chemical composition of the amyloid fibrils (light chain, heavy chain, serum AA protein, insulin, $\beta_{2}$-macroglobulin, fibrinogen, and transrethinin) as well as the pattern of distribution (localized or systemic).

Clinical signs and symptoms are often nonspecific and usually the diagnosis is established by histological detection of amyloid. The pathognomonic sign of amyloid deposition is "apple-green" birefringence within the analyzed tissue or vascular wall. Based on the intensity of the stain, cases can be diagnosed as positive for amyloid, negative for amyloid, or equivocal for amyloid deposition. Results show wide variability between pathologists, with most cases falling within the equivocal zone.

The most common method for histological demonstration of amyloid deposition is the fine-needle aspiration (FNA) of the abdominal fat pad. The procedure is fast, cheap, and relatively noninvasive with minimal side effects. The turnaround time is usually within 24 hours, with results available on the next day. One significant draw back of this procedure is the evaluation of the Congo-red slides under polarized light. Unlike surgical specimens, the polarizable areas in the fat-pad aspirate specimen are usually presented in small foci. It can be 
quite challenging to determine the true color of a small focus of polarizable color. Blue or green that is the question! A critical diagnosis is often based on the answer to this simple dilemma and the answers are frequently subjective based on observation with our naked eye.

In our current study, we propose a fast and reliable method of image analysis as an ancillary study, using commercially available software Adobe Photoshop (C) CS3 Extended Edition.

\section{Materials and Methods \\ Subjects}

The University of Michigan pathology database was searched for all fat biopsy specimens between the years 2004 and 2006. A total of 22 subjects had fat pad biopsies meeting our guidelines for satisfactory specimens (cell block containing mature fibroadipose tissue with at least one blood vessel) and had more than 2-years follow-up in our clinics.

\section{Abdominal Fat Pad Biopsy}

Skin and subcutaneous tissues were anesthetized with lidocaine. Fat tissue, on both sides of the umbilicus in four random areas, was aspirated by trained cytopathologists, with an 18-gauge needle attached to a $10-\mathrm{ml}$ syringe FNA gun. The aim was to aspirate at least $30 \mathrm{mg}$ of fat tissue. The needle is rinsed in Cytolyt $(\mathrm{C}$ and a cell block is prepared from the sediment using the Histo-gel method. The affinity of tissue for Congo-red staining was analyzed by "apple-green" birefringence as observed with polarized light using an Olympus microscope. The adequacy of the biopsy was assessed by examining the hematoxylin and eosin stained cell block slides first. Cases composed with mature fibroadipose tissue and at least one vessel were considered adequate and were subjected to further analysis.

\section{Congo-Red Staining}

Congo-red staining was performed using standard laboratory technique. Briefly, the slides were hydrated in water and placed in Congo-red solution (Sigma, St. Louis, MO) for 5 minutes. The differentiation was done in alcoholic potassium hydroxide solution for 7 dips. The slides were then counterstained with Mayer's hematoxylin for $1 \mathrm{mi}-$ nute, washed with tap water, dehydrated, and mounted.

\section{Image Capture and Analysis Using Adobe Photoshop ${ }^{\circledR}$ CS3 Extended Edition}

The image accusation was done on $400 \times$ magnification, using an Olympus microscope coupled with a Diagnostic Instruments Spot Insight 4 mega pixels CCD camera with automatic exposure time under polarized light. The images were saved in uncompressed (TIFF) format for future analysis. The saved images were opened in Adobe Photoshop(C) CS3 Extended edition and the areas of inter-
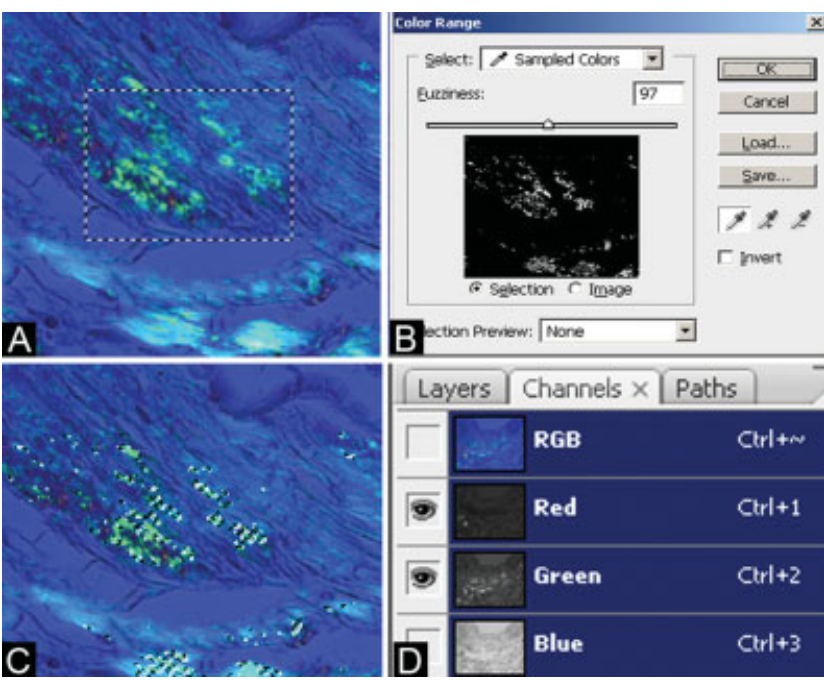

Fig. C-1. Step-by-step algorithm for obtaining the blue:green ratio in Adobe photoshop CS3.

est were selected by using the Rectangular Marquee tool. Multiple areas were selected by holding down the SHIFT key when selecting different areas. Once the areas were selected from the menu, we proceeded with choosing the Select from the tool bar and then Color Range option from the drop down menu. With the Eye Dropper tool, the color of interest within the selected areas was chosen and fuzziness of the selection was adjusted to cover maximum area of the color of interest and decrease the background. A proper control of the selected areas can be established by switching the radio buttons between the Selection and Image options. The fussiness (size of selected region) of the interested area was at 80 , which was validated. Once a satisfactory result is obtained, clicking the OK button will result in further color selection, which includes only the color of interest. The analysis was performed by switching off the Blue channel first and then selecting the Analysis menu, and then Record Measurements calculates the green channel mean gray value. With the selection still active, the Blue channel was switched on and the Green channel was switched off. The measurements were recorded again using Analysis and then Record Measurements option. All the measurements were exported as text file (Fig. C-1).

\section{Data Analysis}

Data analysis was done using Microsoft Excel software. The measurements recorded with Adobe Photoshop were imported in Microsoft Excel. For the purpose of this study we used only values of the Mean Gray color. All the values of the Green and Blue channel were averaged, and 
Table I. Changing the Fuzziness of the Selected Areas Results in Increase or Decrease of the Total Number of Selected Areas

\begin{tabular}{|c|c|c|c|c|}
\hline Fuzziness & 20 & 40 & 60 & 80 \\
\hline $\begin{array}{l}\text { G/B mean } \\
\text { gray color }\end{array}$ & $2.96 \pm 0.11$ & $2.98 \pm 0.09$ & $2.97 \pm 0.07$ & $2.95 \pm 0.08$ \\
\hline $\begin{array}{l}\text { Total selected } \\
\text { areas }\end{array}$ & 110 & 49 & 32 & 19 \\
\hline
\end{tabular}

The change of fuzziness does not change the blue:green ratio.

the ratio was calculated by dividing Green by the Blue channel. The resulting number was called amyloid number (AN). The AN of the analyte was compared to its representative positive control using single factor ANOVA analysis following the standard Excel routines. If the observed $P$ value is less that 0.05 (statistically significant difference) and the AN of the positive control is bigger than that of the analyte, the specimen is labeled as negative for amyloid. Respectively, if there is no significant difference between the analyte and its positive control, the specimen was labeled positive for amyloid.

\section{Results}

For Adobe Photoshop(C, all colors are coded in black and white. When using the RGB mode, every pixel on the screen is represented by three separate channels Red, Green, and Blue. Each of these channels has a value of $0-255$ where 0 is black and 255 is white. Depending on how much light goes through the filters, the color of each pixel on the screen is calculated based on combination of the earlier three channels. For example, the color yellow is a result of mixing of red and green. To represent this color intensity on the screen, the software will shine light through the red filter (channel) first. If all of the light goes through the filter, the value of that channel will be set to 255 or white. If no light is able to pass through the channel, the value will be set to 0 (or black). Same process will repeat for all three filters. Thus to represent the yellow color, the software will use a combination of filters with values: R:255 G:255: B:0. The accuracy of the color will depend on the color resolution of the monitor. Most modern computer monitors will represent 16 million colors; thus, the images will contain information coded in shades of gray color. These shades of gray can be calculated by turning off the different filters (channels) and analyzing the mean gray color of any selected area.

\section{Validation of the Digital Analysis Using Positive and Negative Controls}

One of the difficulties in evaluating the Congo-red stained fat pad biopsies arises from the linear stain and birefringence of the collagen bundles. Our first experiments were directed toward discriminating the blue-green collagen birefringence from amyloid "apple-green" birefringence
Table II. The Negative Controls From Heart, Skin, and Fibrous Tissues Show Statistically Significant Difference From the Positive Amyloid Control

\begin{tabular}{lcccc}
\hline & Amyloid & Heart & Skin & Fibrous tissue \\
\hline $\begin{array}{l}\text { Total areas } \\
\text { Blue:green }\end{array}$ & 100 & 134 & 291 & 184 \\
$\quad$ AN & $3.55 \pm 0.23$ & $1.55 \pm 0.09$ & $1.93 \pm 0.15$ & $1.69 \pm 0.1$ \\
$P$ value & - & $1.9 \mathrm{E}-181$ & $8.1 \mathrm{E}-240$ & $3.6 \mathrm{E}-211$ \\
\hline
\end{tabular}

observed and analyzed under the same conditions. For the Photoshop image analysis, we used sections from a normal heart muscle tissue, fibrous tissues (scar), and skin samples stained with Congo-red. One positive amyloid control was also included. The image analysis was done as described in Materials and Methods. There is an unavoidable bias when selecting the colors for analysis and that includes the inability of the evaluator to accurately discriminate by eye between the "apple-green" birefringence and blue-green collagen birefringence. Another variable when selecting a color for analysis is the fuzziness of the selection. Fuzziness determines the tightness or the amount of color selected. In this respect, the lower the number the less color areas will be defined. This can be illustrated by measuring the same areas with different fuzziness (Table I). This measurement shows that increasing the fuzziness will decrease the number of selected areas; however, the blue/green ratio will be preserved. In our experiments, we found that fuzziness between 60 and 80 gives the optimal number of selected areas between 100 and 300 per analyte. The observed measurements were compared using single-parameter ANOVA analysis. All of the analytes showed that the $P$ values are statistically significant from the positive amyloid control, which demonstrates the ability of the test to discriminate between collagen stain versus amyloid stain (Table II). In this analysis, a cutoff level of 3.0 successfully identified an amyloid case from the representative negative controls (Fig. 1).

\section{Discrimination Between Blue and "Apple-Green" Areas Within the Same Image}

In order to investigate the ability of the software to discriminate between true blue and true "apple-green" areas, we analyzed over 700 different areas within the same positive control for amyloid. The areas were chosen by using Select from the menu and then Color range. Using the picker, the proper blue or "apple-green" colors were selected. The individual measurements were done as described in Materials and Methods. More than 300 areas were analyzed. The distance between the blue and green areas depends on the fuzziness of the selection. The higher the fuzziness number is the closer both areas will be due to decreased tightness of the selection. In this experiment, the fuzziness was kept at 75 , which showed 


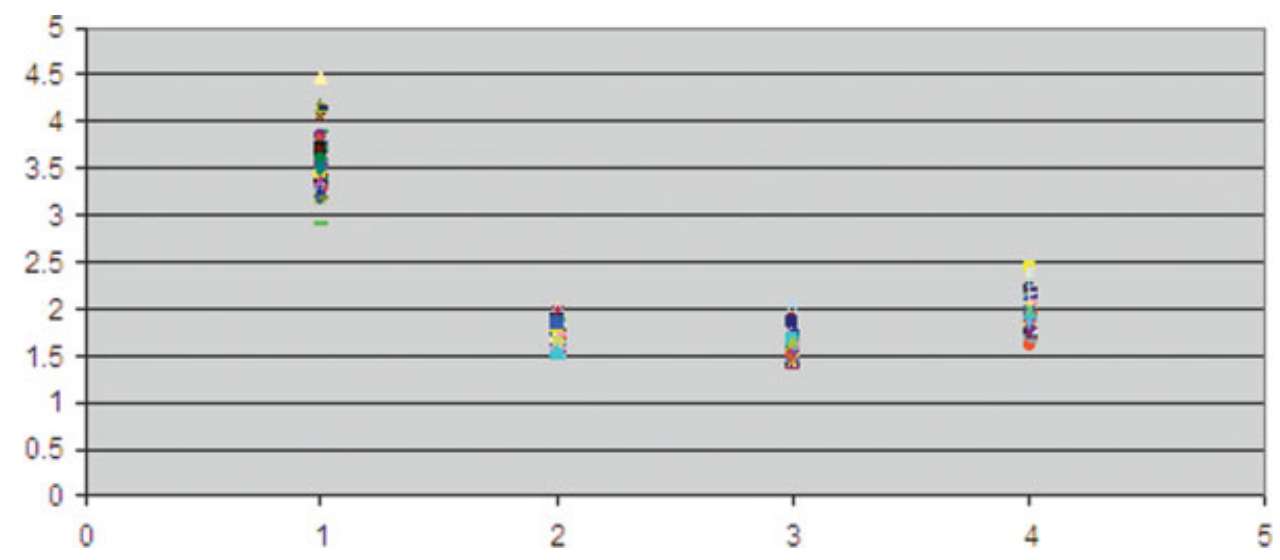

Fig. 1. Distribution of the selected areas of the negative control in comparison with the representative positive control. 1: Amyloid positive control, 2: Skin, 3: Fibrous tissue, 4: Heart muscle. [Color figure can be viewed in the online issue, which is available at www.interscience.wiley.com.]

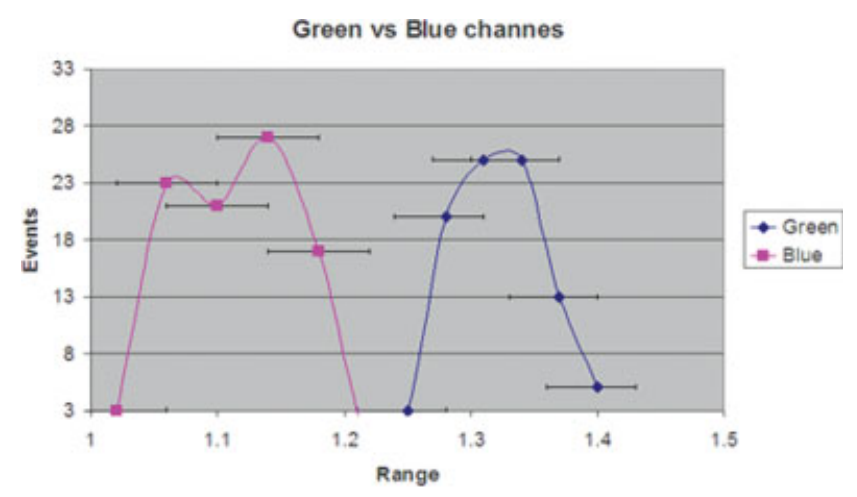

Fig. 2. Histogram plot of blue: green ratios of blue and green areas within the same control positive for amyloid. [Color figure can be viewed in the online issue, which is available at www.interscience.wiley.com.]

the best separation. The histogram plots of both areas are shown on Figure 2.

\section{Image Analysis of Clinical Fat Pad Biopsies}

For the purpose of our study, we have followed up 22 patients who were referred to the FNA clinic at the Cancer Center at the University of Michigan for a fat pad biopsy between year 2004 and 2006. The median age of the patients was 61 years, and the male-to-female ratio was 13:9. The most common disease entities as well as the patient demographics are shown in (Table III). Two cytopathologists unaware of the patient histories evaluated all the cases and have classified them as positive, negative, or equivocal. All the cases were subjected to a computerassisted analysis, and each case was compared to its matched control.

At least 100 different areas per image were analyzed, and the ratio between the green and blue channel was obtained as described in the Materials and Methods. If there was no statistical significance between the analyte and its control, the case was labeled as positive, and if there was a statistically significant difference between the analyte and the control and the analyte had a score lower than the control, the case was labeled as negative.

In this study, the fat pad biopsies of 16 patients were originally interpreted as negative from which two (cases 3 and 20) have developed systemic amyloidosis on a 2-year follow-up. Computer analysis of these 2 patients showed that the biopsy of patient number three had no statistical difference from its control $(P=0.7)$ and thus was interpreted as positive. The patient number 20 tested consistently negative either with computer analysis or by slide review by two pathologists (SB and YP). This case most likely represents a false-negative case, which is inherent to sampling rather than interpretational analysis. The remaining 6 cases out of 22 were signed out as positive. From these cases, three patients numbered 8, 19 and 21 have developed a systemic amyloidosis on a follow-up; thus, the interpretations were considered true positive results. Cases 8 and 19 were confirmed with image analysis to be truly positive; however, case 21 had repeatedly tested negative by computer analysis. The slide review by two pathologists (SB and RP) showed it to be positive for amyloid; thus, this case represents a false-negative by image analysis. The specificity and sensitivity of the computer-assisted image analysis were calculated to be $75 \%$ and $100 \%$, respectively. All three patients with proven systemic amyloidosis were correctly identified by the computer. The slide review showed a sensitivity and specificity of $50 \%$ and $87 \%$, respectively. These results are in agreement with the published papers; however, caution should be exercised before drawing firm conclusions because of the small sample size presented here.

\section{Discussion}

The diagnosis of amyloidosis is established by demonstration of the abnormal fibrilary depositions in tissue biopsy specimens usually from abdominal subcutaneous fat. The procedure is called random fat pad needle biopsy and is 
BARDAROV ET AL.

Table III. Patients and Analysis of Fat Pad Biopsies

\begin{tabular}{|c|c|c|c|c|c|c|c|c|c|}
\hline & Sex & Age & Analyte & Cont & $P$ value & Interpret & Clinical & SO & $D X$ \\
\hline 1 & M & 75 & 1.70 & 1.74 & $5.72 \mathrm{E}-05$ & Negative & No amyloidosis & Negative & MGUS \\
\hline 2 & M & 60 & 1.81 & 1.85 & 0.04 & Negative & No amyloidosis & Negative & MGUS \\
\hline 3 & M & 62 & 1.77 & 1.77 & 0.7 & Positive & Renal amyloidosis & Negative & MM \\
\hline 4 & $\mathrm{M}$ & 68 & 1.61 & 1.68 & $4.30 \mathrm{E}-07$ & Negative & No amyloidosis & Negative & MGUS \\
\hline 5 & M & 70 & 1.59 & 1.68 & $3.74 \mathrm{E}-11$ & Negative & No amyloidosis & Negative & MGUS \\
\hline 6 & $\mathrm{~F}$ & 60 & 1.7 & 2.33 & $1.60 \mathrm{E}-68$ & Negative & No amyloidosis & Positive & Diabetes \\
\hline 7 & $\mathrm{~F}$ & 46 & 1.93 & 2.3 & $5.30 \mathrm{E}-45$ & Negative & No amyloidosis & Negative & Diabetes \\
\hline 8 & M & 63 & 1.69 & 1.66 & 0.000977 & Positive & Amyloidosis & Positive & MM \\
\hline 9 & M & 58 & 1.27 & 1.68 & $2.26 \mathrm{E}-77$ & Negative & No amyloidosis & Negative & MM \\
\hline 10 & $\mathrm{~F}$ & 68 & 0.98 & 1.67 & $1 \mathrm{E}-127$ & Negative & No amyloidosis & Negative & MGUS \\
\hline 11 & $\mathrm{~F}$ & 59 & 1.06 & 1.75 & $2.18 \mathrm{E}-45$ & Negative & No amyloidosis & Negative & RA \\
\hline 12 & M & 60 & 1.09 & 1.55 & $1 \mathrm{E}-202$ & Negative & No amyloidosis & Negative & MGUS \\
\hline 13 & M & 58 & 1.04 & 1.31 & $1 \mathrm{E}-202$ & Negative & No amyloidosis & Negative & MGUS \\
\hline 14 & $\mathrm{~F}$ & 83 & 1.02 & 1.1 & $\ll 0.005$ & Negative & No amyloidosis & Negative & MM \\
\hline 15 & $\mathrm{~F}$ & 62 & 1.1 & 1.2 & $\ll 0.05$ & Negative & No amyloidosis & Negative & RA \\
\hline 16 & $\mathrm{~F}$ & 46 & 0.97 & 1.1 & $\ll 0.06$ & Negative & No amyloidosis & Negative & MM \\
\hline 17 & $\mathrm{~F}$ & 52 & 1.9 & 2.4 & $5.00 \mathrm{E}-39$ & Negative & No amyloidosis & Positive & MGUS \\
\hline 18 & M & 76 & 1.86 & 2.2 & $1.50 \mathrm{E}-24$ & Negative & No amyloidosis & Negative & MM \\
\hline 19 & $\mathrm{~F}$ & 66 & 2.1 & 1.9 & $9.30 \mathrm{E}-65$ & Positive & Amyloidosis cardiac & Positive & MM \\
\hline 20 & M & 48 & 2.07 & 2.3 & $1.43 \mathrm{E}-04$ & Negative & Amyloidosis & Negative & Hep C \\
\hline 21 & M & 42 & 1.67 & 2 & $2.00 \mathrm{E}-03$ & Negative & Amyloidosis & Positive & MGUS \\
\hline 22 & M & 54 & 1.7 & 2 & $1.00 \mathrm{E}-02$ & Negative & No amyloidosis & Positive & RA \\
\hline
\end{tabular}

MGUS, monoclonal gammopathy of unknown significance; MM, multiple myeloma; RA, rheumatoid arthritis; Hep C, hepatitis C; SO, signed out as.

performed by trained cytopathologists in clinical settings. The obtained tissue is stained with Congo-red for which the amyloid has been proven to have high affinity and then examined under polarized light for "apple-green" birefringence. Other techniques for tissue procurement and examination like rectal biopsy or organ needle biopsy are considered invasive with increased morbidity and are generally more invasive.

One of the problems when examining tissue for amyloid under polarized light is the variability in interpretation among different pathologists performing the examination, thus giving a high rate of false-negative or false-positive results. In one study, Guy and Jones ${ }^{1}$ examined 45 samples over a 3-year period. Seven patients had a clinical diagnosis of amyloidosis that tested positive for a Congo-red stain, giving an excellent specificity of $100 \%$. The sensitivity however was calculated to be rather low (58\%), and the inadequacy was measured to be about $11 \%$. In a similar study, Ansari-Lari and Ali have addressed the issue of the clinical usefulness of the fat pad biopsy. ${ }^{2}$ In this study, they followed up 91 patients who underwent abdominal fat pad biopsy for the diagnosis of amyloid. The sensitivity was calculated to be $55 \%$, similar to the one observed by Guy and Jones, and the specificity was shown to be $75 \%$. In the same study follow-up biopsies were not available, suggesting that the clinical picture is in agreement with the pathological finding and when added to the rest of the cases the specificity and sensitivity increased to $75 \%$ and $92 \%$, respectively. In another study, Hazenberg et al estimated a sensitivity of $80 \%$ and specificity of $100 \%$ by following up 120 patients. $^{3}$ The sensitivity increased to $93 \%$ when the specimens were thoroughly examined. Similar conclusion was reached by Dhingra et al. ${ }^{4}$ who concluded that abdominal fat pad aspirations are useful for the diagnosis of systemic amyloidosis with sensitivities and specificities of $78 \%$ and $93 \%$, respectively.

Today there are additional tools available for analysis of mature adipose tissue aspirates for amyloidosis and they include fluorescent microscopy, immunohistochemistry and electron microscopy; however, these studies require additional techniques and trained personal, and ultimately increase the cost of this diagnostic procedure.

One of the pitfalls in the interpretation of the stain is the false positivity because of a linear and parallel birefringence of collagen fibers. On the other hand, improper staining protocols, improper controls, or improper use of polarizing equipment can lead to false-negative interpretations.

In this study, we present a methodology and preliminary result on the usefulness of the computer-assisted image analysis in the diagnosis of amyloidosis in fat pad biopsies. The methodology correctly discriminated between the true amyloid and the blue-green birefringence of the collagen bundles. It also correctly identified a subset of patients with clinically proven amyloidosis. This analysis must be prospectively assessed and validated in a study with a larger sample size, tight quality control of the staining technique, and strict patient follow-up before implementing it in routine practice. Our data suggests that this methodology may be useful in the evaluation of amyloidosis in fat pad biopsies or it can serve as an additional confirmation analysis in questionable specimens. The advantage of this methodology is that it can be carried 


\section{AMYLOID DEPOSITS IN ABDOMINAL FAT PAD ASPIRATION BIOPSIES}

out on slides already stained with Congo-red and it does not require any additional tissue processing, thus there is no need of implementing new technique in the surgical pathology laboratory nor will it cause delay in the turnaround times. Adobe PhotoshopC CS3 is available in pathology labs or can be purchased for a reasonable price. No special personnel training are necessary when performing the above analysis. All results need to be interpreted in the parallel with the clinical picture. To the best of our knowledge, this is the first report that describes the use of commercially available software Adobe Photoshop CS3 in analysis of amyloid deposits in soft tissues.

\section{References}

1. Guy CD, Jones CK. Abdominal fat pad aspiration biopsy for tissue confirmation of systemic amyloidosis: Specificity, positive predictive value, and diagnostic pitfalls. Diagn Cytopathol 2001;24:181-185.

2. Ansari-Lari MA, Ali SZ. Fine-needle aspiration of abdominal fat pad for amyloid detection: A clinically useful test? Diagn Cytopathol 2004;30:178-181.

3. Hazenberg BP, Bijzet J, van Rijswijk MH. Diagnostic accuracy of subcutaneous abdominal fat tissue aspiration for detecting systemic amyloidosis and its utility in clinical practice. Arthritis Rheum 2006; 54:2015-2021.

4. Dhingra S, Krishnani N, Kumari N, Pandey R. Evaluation of abdominal fat pad aspiration cytology and grading for detection in systemic amyloidosis. Acta Cytol 2007;51:860-864. 